\title{
Radiation damage in protein serial femtosecond crystallography using an x-ray free-electron laser
}

Lukas Lomb, ${ }^{1,2}$ Thomas R. M. Barends, ${ }^{1,2}$ Stephan Kassemeyer,,${ }^{1,2}$ Andrew Aquila, ${ }^{3}$ Sascha W. Epp,,${ }^{2,4}$ Benjamin Erk, ${ }^{2,4}$ Lutz Foucar, ${ }^{1,2}$ Robert Hartmann, ${ }^{5}$ Benedikt Rudek,${ }^{2,4}$ Daniel Rolles, ${ }^{1,2}$ Artem Rudenko, ${ }^{2,4}$ Robert L. Shoeman, ${ }^{1,2}$ Jakob Andreasson, ${ }^{6}$ Sasa Bajt, ${ }^{7}$ Miriam Barthelmess, ${ }^{7}$ Anton Barty, ${ }^{3}$ Michael J. Bogan, ${ }^{8}$ Christoph Bostedt, ${ }^{9}$ John D. Bozek, ${ }^{9}$ Carl Caleman, ${ }^{3}$ Ryan Coffee, ${ }^{9}$ Nicola Coppola,,${ }^{3, *}$ Daniel P. DePonte, ${ }^{3}$ R. Bruce Doak, ${ }^{10}$ Tomas Ekeberg, ${ }^{6}$

Holger Fleckenstein, ${ }^{3}$ Petra Fromme, ${ }^{11}$ Maike Gebhardt, ${ }^{1}$ Heinz Graafsma, ${ }^{7}$ Lars Gumprecht, ${ }^{3}$ Christina Y. Hampton, ${ }^{8}$ Andreas Hartmann, ${ }^{5}$ Günter Hauser, ${ }^{12,13}$ Helmut Hirsemann, ${ }^{7}$ Peter Holl, ${ }^{5}$ James M. Holton, ${ }^{14}$ Mark S. Hunter,,${ }^{11}$

Wolfgang Kabsch, ${ }^{1}$ Nils Kimmel, ${ }^{12,13}$ Richard A. Kirian, ${ }^{10}$ Mengning Liang, ${ }^{3}$ Filipe R. N. C. Maia, ${ }^{6,15}$ Anton Meinhart, ${ }^{1}$ Stefano Marchesini, ${ }^{14}$ Andrew V. Martin, ${ }^{3}$ Karol Nass, ${ }^{16}$ Christian Reich, ${ }^{5}$ Joachim Schulz, ${ }^{3}$ M. Marvin Seibert, ${ }^{6,9}$ Raymond Sierra, ${ }^{8}$ Heike Soltau, ${ }^{5}$ John C. H. Spence, ${ }^{10}$ Jan Steinbrener, ${ }^{1,2}$ Francesco Stellato, ${ }^{3}$ Stephan Stern, ${ }^{3}$

Nicusor Timneanu, ${ }^{6}$ Xiaoyu Wang, ${ }^{10}$ Georg Weidenspointner, ${ }^{12,13}$ Uwe Weierstall, ${ }^{10}$ Thomas A. White, ${ }^{3}$ Cornelia Wunderer, Henry N. Chapman, ${ }^{3,16}$ Joachim Ullrich, ${ }^{2,4}$ Lothar Strüder, ${ }^{2,12}$ and Ilme Schlichting ${ }^{1,2, \dagger}$

${ }^{1}$ Max-Planck Institut für medizinische Forschung, Jahnstrasse 29, DE-69120 Heidelberg, Germany

${ }^{2}$ Max Planck Advanced Study Group, Center for Free-Electron Laser Science, Notkestrasse 85, DE-22607 Hamburg, Germany

${ }^{3}$ Center for Free-Electron Laser Science, DESY, Notkestrasse 85, DE-22607 Hamburg, Germany

${ }^{4}$ Max-Planck Institut für Kernphysik, Saupfercheckweg 1, DE-69117 Heidelberg, Germany

${ }^{5}$ PNSensor GmbH, Otto-Hahn-Ring 6, DE-81739 München, Germany

${ }^{6}$ Laboratory of Molecular Biophysics, Department of Cell and Molecular Biology, Uppsala University, Husargatan 3 (Box 596), SE-751 24 Uppsala, Sweden

${ }^{7}$ Photon Science, DESY, Notkestrasse 85, DE-22607 Hamburg, Germany

${ }^{8}$ Stanford PULSE Institute, SLAC National Accelerator Laboratory, 2575 Sand Hill Road, Menlo Park, California 94025, USA

${ }^{9}$ Linac Coherent Light Source, SLAC National Accelerator Laboratory, 2575 Sand Hill Road, Menlo Park, California 94025, USA

${ }^{10}$ Department of Physics, Arizona State University, Tempe, Arizona 85287, USA

${ }^{11}$ Department of Chemistry and Biochemistry, Arizona State University, Tempe, Arizona 85287, USA

${ }^{12}$ Max-Planck-Institut Halbleiterlabor, Otto-Hahn-Ring 6, DE-81739 München, Germany

${ }^{13}$ Max-Planck-Institut für extraterrestrische Physik, Giessenbachstrasse, DE-85741 Garching, Germany

${ }^{14}$ Advanced Light Source, Lawrence Berkeley National Laboratory, Berkeley, California 94720, USA

${ }^{15}$ National Energy Research Scientific Computing Center, Lawrence Berkeley National Laboratory, Berkeley, California 94720, USA

${ }^{16}$ University of Hamburg, Luruper Chaussee 149, DE-22761 Hamburg, Germany

(Received 23 July 2011; revised manuscript received 19 October 2011; published 20 December 2011)

\begin{abstract}
$\mathrm{X}$-ray free-electron lasers deliver intense femtosecond pulses that promise to yield high resolution diffraction data of nanocrystals before the destruction of the sample by radiation damage. Diffraction intensities of lysozyme nanocrystals collected at the Linac Coherent Light Source using $2 \mathrm{keV}$ photons were used for structure determination by molecular replacement and analyzed for radiation damage as a function of pulse length and fluence. Signatures of radiation damage are observed for pulses as short as $70 \mathrm{fs}$. Parametric scaling used in conventional crystallography does not account for the observed effects.
\end{abstract}

The elucidation of macromolecular structures is an important step in the quest for understanding the chemical mechanisms underlying biological function. X-ray crystallography is a mature yet still advancing method that allows structure determination of molecules with any molecular weight. The ultimate structural resolution is limited only by the crystal quality (size and order of the lattice) and by radiation damage. In many cases, growing large crystals is extremely challenging-in particular for large macromolecular complexes and membrane proteins. Crystal size and radiation damage are entangled entities; a reduction in crystal size demands for higher fluence to sustain sufficient diffraction intensity, ${ }^{1}$ increasing the extent of radiation damage.

Initial radiation damage inflicted by hard $\mathrm{x}$-rays in the energy range up to about $20 \mathrm{keV}$ is dominated by the photoelectric effect. Removing electrons from individual atoms leads to electronic ${ }^{2-5}$ and chemical changes ${ }^{6-9}$ and will ultimately decrease the overall scattering power of the sample. Crystals cooled to liquid nitrogen temperature tolerate a dose of $30 \mathrm{MGy}{ }^{10}$ It has been predicted that this dose ${ }^{10,11}$ can be increased significantly if the diffraction data is collected using intense femtosecond $\mathrm{x}$-ray pulses, which are short enough to have passed the sample before significant electronic rearrangements and atomic displacements occur ("diffractionbefore-destruction"12). The advent of free-electron lasers (FELs) provides femtosecond pulses with peak brilliances that are about nine orders of magnitude higher than those provided by third generation synchrotron sources. The newly commissioned FEL at SLAC National Accelerator Laboratory, the Linac Coherent Light Source (LCLS), ${ }^{13}$ accesses the hard-x-ray regime. Recently, the concept of serial femtosecond crystallography (SFX) has been demonstrated on photosystem I (PSI) by injecting a stream of hydrated nanocrystals into the FEL interaction region, collecting diffraction patterns from single, randomly oriented crystals hit by the femtosecond x-ray pulses. ${ }^{14}$ In SFX, due to the high intensity of the FEL, the crystal is destroyed and has to be replaced after each exposure. 
TABLE I. FEL beam parameters, absorbed dose, and Blake- and Phillips-type Wilson scaling factors. The FEL pulse energies were measured in the beamline gas detector. The pulse fluence was calculated assuming $25 \%$ beamline transmission and a $10 \mu \mathrm{m}^{2}$ focus.

\begin{tabular}{lcccccccc}
\hline \hline Pulse length (fs) & 70 & 85 & 100 & 150 & 200 & 250 & 300 & 400 \\
\hline Pulse energy (mJ) & 2.11 & 2.25 & 2.32 & 2.71 & 2.28 & 1.29 & 0.96 & 0.47 \\
Pulse intensity (PW/cm $\left.{ }^{2}\right)$ & 75.7 & 65.9 & 58.0 & 45.3 & 28.5 & 12.8 & 8.0 & 4.0 \\
Pulse fluence $\left(\mathrm{kJ} / \mathrm{cm}^{2}\right)$ & 5.3 & 5.6 & 5.8 & 6.8 & 5.7 & 3.2 & 2.4 & 1.2 \\
Dose $(\mathrm{GGy})^{\mathrm{b}}$ & 2.4 & 2.6 & 2.7 & 3.1 & 2.6 & 1.5 & 1.1 & 0.5 \\
Wilson $k$ & 3.9 & 5.2 & 4.9 & 4.6 & 4.3 & 4.0 & 4.4 & 3.5 \\
Wilson $B\left(\AA^{2}\right)$ & 109 & 107 & 111 & 146 & 155 & 178 & 182 & 141 \\
\hline \hline
\end{tabular}

$\overline{\bar{a}}$ All pulse lengths given here are nominal pulse lengths as extracted from machine data. X-ray-pulse durations may differ from these values due to the statistical characteristics and highly collective process of the self-amplified spontaneous-emission effect. It has been shown recently that the $\sim 80$-fs mode of operation in fact produces $x$-rays of shorter duration ${ }^{3}$

${ }^{\mathrm{b}}$ Absorbed by an isolated crystal. The doses absorbed by crystals and the surrounding water jet are $3.4,3.7,3.8,4.4,3.7,2.1,1.6,0.8$, and 2.9 GGy, respectively.

Here, we quantitatively analyze the quality of such data as a function of incident irradiance and FEL pulse length using lysozyme nanocrystals ${ }^{15}$ as a model system. The experiments were performed at the $\operatorname{LCLS}^{13}$ at the Atomic Molecular and Optical (AMO) beamline ${ }^{16}$ in vacuo in the CFEL-ASG Multi-Purpose (CAMP) instrument. ${ }^{17}$ Using a liquid microjet, ${ }^{18}$ chemically cross-linked, monodisperse lysozyme crystals $\left(0.4 \times 0.4 \times 0.8 \mu \mathrm{m}^{3}\right)^{19}$ immersed in a continuous stream of water that was $4 \mu \mathrm{m}$ in diameter were introduced into the spatially coherent, monochromatic FEL beam. The maximum photon energy available at the AMO beamline ( $2 \mathrm{keV}, 6.2 \AA$ wavelength) was used with nominal pulse durations between $70 \mathrm{fs}$ and $400 \mathrm{fs}$ and more than $10^{12}$ photons/pulse (Table I). The pulses were focused to a $10 \mu \mathrm{m}^{2}$ spot. ${ }^{13}$ The crystals absorbed a dose of up to $3 \mathrm{GGy}$ each (Table I) as calculated with the program RADDOSE. ${ }^{20}$ Single-shot diffraction data were recorded using a pair of pnCCD detectors (Fig. 1), ${ }^{14,17}$ operating at the $60 \mathrm{~Hz}$ repetition rate of the FEL. Detector halves were placed $66.0 \mathrm{~mm}$ and $68.6 \mathrm{~mm}$ downstream of the interaction region, covering angles between $4.1^{\circ}$ to $48^{\circ}$.

Offset- and common-mode corrections were applied to reduce the detector-based background in the diffraction patterns. ${ }^{14}$ Bragg peaks in the individual diffraction patterns were identified and located by applying a discrimination algorithm based on a local threshold of four standard deviations above the mean. The local background around each peak was subtracted using a method similar to that implemented in DENZO. ${ }^{21}$ Peak positions were corrected with respect to pulse-to-pulse wavelength variations in every individual diffraction pattern. After polarization correction, assuming 99\% polarization in the horizontal plane, the indexing of diffraction patterns was reduced to a one-dimensional problem by combining the integrated Bragg peaks into a virtual powder pattern for each pulse length [Figs. 2(a) and 2(b)]. This is possible for lysozyme because individual rings were well-separated on the detector. The resulting Debye-Scherrer rings are characterized by their Bragg angle $2 \theta$ and intensities $I$. The radially integrated one-dimensional intensity profiles [Fig. 2(c)] were modeled as a sum of pseudo-Voigt profiles.

The intensities obtained were limited to $7.6 \AA$ resolution by the x-ray wavelength and the geometry of the experimental setup. The quality of the SFX-derived diffraction intensities was analyzed by comparison with conventional powder diffraction data collected at $10 \mathrm{keV}$ at the Swiss Light Source from crystals of the same batch used in the SFX experiments. The correlation coefficients between intensities extracted by curve fitting from the SFX data and conventional powder diffraction data were between $84 \%$ and $93 \%$ for all pulse lengths to $10 \AA$ resolution after which the correlations degraded due to overlapping Debye-Scherrer rings. To analyze the quality of the SFX-derived data with respect to structure determination, we phased the SFX diffraction intensities by molecular replacement. A lysozyme model (PDB entry 1 VDS) was randomly oriented and positioned in space, and a rotation and translation search was performed with the CCP4 program FSEARCH. ${ }^{22}$ Diffraction data of all pulse lengths yielded molecular replacement solutions that were in

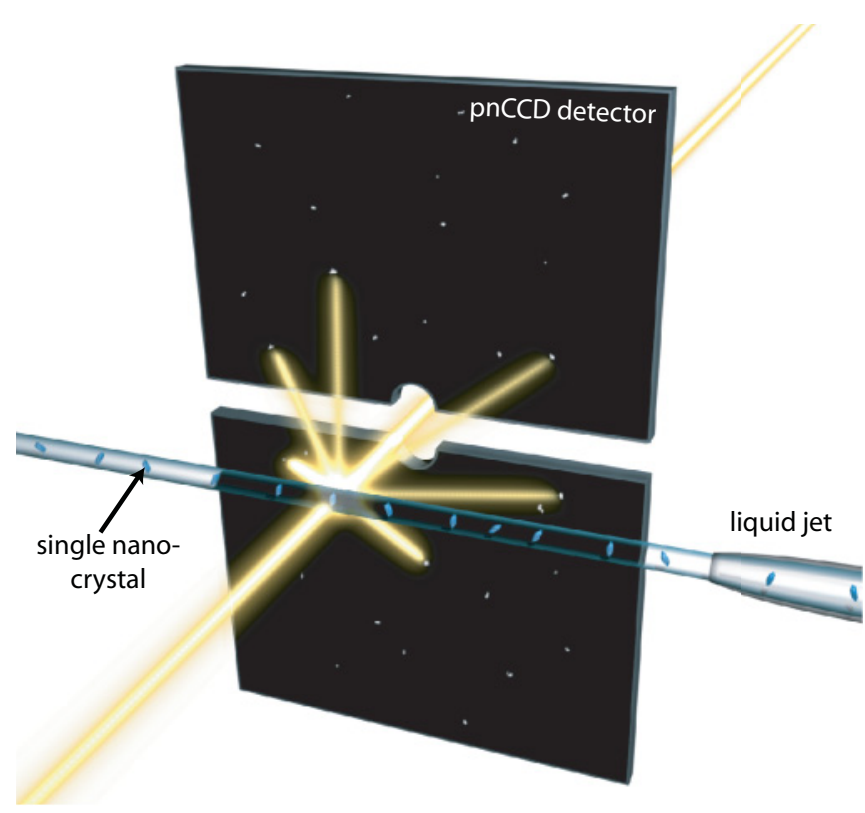

FIG. 1. (Color) Experimental setup. Randomly oriented lysozyme nanocrystals (blue) were introduced into the focus of the x-ray beam (yellow) by a gas-focused water jet (gray) (Ref. 18). Diffraction data (white spots) were collected using a pair of pnCCD detectors (black) (Refs. 14 and 17), set at different vertical distances with respect to the beam to cover high scattering angles. 


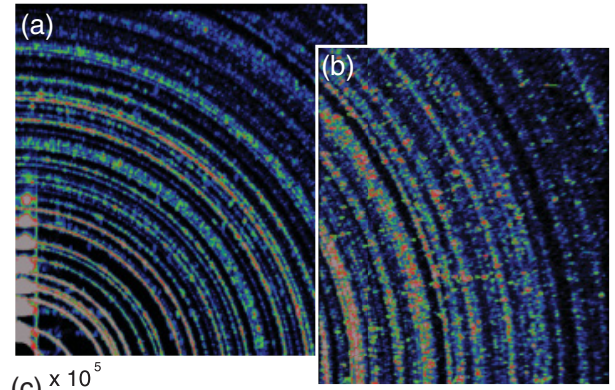

(c) $\times 10$
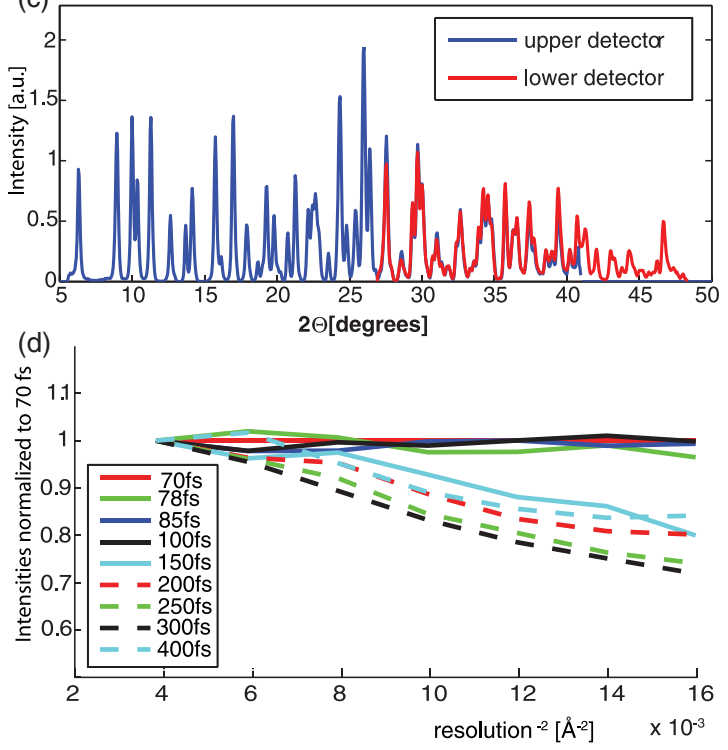

FIG. 2. (Color) Virtual powder diffraction data collected on a pair of pnCCD detectors and comparison of intensities for the different pulse durations. (a) Section of the virtual powder pattern (70 fs SFX data) calculated for the upper pnCCD detector. Scattering of the liquid jet column containing the nanocrystals produces the vertical streak shown in the lower-left corner. (b) Section of the lower detector. Intensities are scaled by a factor of 3 for better visualization. (c) Transformation of the upper (blue) and lower (red) detector data into a 1-dimensional intensity plot. (d) Radiation damage at different pulse durations analyzed by comparing scattered intensities for different resolution bins. Intensities were averaged by moving a sliding window over the 1-dimensional powder patterns. All intensities were normalized in the low resolution range $(0.002$, $0.006 \AA^{-2}$ ) and compared to the intensities of the 70 -fs data. Very low resolution data is affected by stray light from the liquid [gray vertical dots in (a)] and were therefore omitted from the analysis.

excellent agreement with the true orientation and position of the lysozyme molecule in the asymmetric unit, demonstrating that structure determination using SFX is feasible (Ref. 23, Fig. S2).

Radiation damage processes start on the femtosecond time scale and may interfere with structure determination. In order to explore the influence of the pulse duration on the diffraction intensities, SFX data were collected using nominal pulse durations (Table I) of 70, 85, 100, 150, 200, 250, 300, $400 \mathrm{fs}$ and $78 \mathrm{fs}$ at the end to rule out systematic changes during the experiment. To enable a meaningful comparison of the various data sets, the effects of variations in the absolute number of crystals exposed in individual runs as well as in FEL pulse energy (Table I) were removed by normalizing all data sets to each other in the low resolution region $(22-13 \AA)$. This region is considered to be least affected by radiation damage and by photons scattered by the surrounding liquid jet, which affect the lowest scattering angles and could not fully be suppressed during the peak-finding process. Ignoring variations in irradiance for the various runs (Table I), we first analyzed the quality of the data as a function of pulse length relative to the $70 \mathrm{fs}$ data, assumed to be the least affected by damage. We found a systematic decrease of the intensities of high resolution reflections for pulse lengths longer than $100 \mathrm{fs}$ [Fig. 2(d)]. The reduced apparent damage of the 400 fs data compared to that of the 300 fs data may be explained by the lower pulse energy of the $400 \mathrm{fs}$ pulses.

The influence of irradiance could be observed not only by changing the beam parameters but also by analyzing the apparent intensity differences between individual diffraction patterns. The uniform size distribution of the crystals established by both TEM and by the Lorentzian shape of the diffraction peaks in the one-dimensional intensity profiles suggested that variation in the crystals was not the source of the observed intensity differences. Therefore, we assumed that high intensities were caused by diffraction from crystals that were located in the center of the approximately Gaussian FEL beam intensity profile and/or were hit by an intense FEL pulse. Indeed, using a subset of the data for all pulse lengths that had similar intensities for the highest intensity diffraction patterns of the $400 \mathrm{fs}$ data showed that the $400 \mathrm{fs}$ data set was damaged to a similar extent as the 300 fs data set (Ref. 23, Fig. S4). To analyze the irradiance dependence of the data, diffraction patterns of each pulse duration were grouped into five classes according to their total diffracted intensities. $^{23}$ The analysis shown in Fig. 2(d) was repeated for each of the five classes, and the results are shown in Fig. 3. Comparison of the resolution-dependent falloff of the different intensity classes shows that the highest-intensity data display the strongest decrease of Bragg intensity versus resolution with increasing pulse length. Accordingly, for weaker diffraction patterns, the falloff at longer pulse lengths is less pronounced, indicating a stronger contribution of less damaged crystals as expected in the weaker beam periphery and/or for lower pulse energies in agreement with models. ${ }^{24}$ Importantly, the analysis of the diffraction patterns collected with 70 fs pulses showed a steeper decrease with resolution for high intensity diffraction patterns compared to the lowest-intensity patterns, indicating the presence of damage even in the 70 fs pulse length data (Fig. 3).

The decay of the diffraction intensity as a function of resolution and dose is an effect that conventional crystallographic data-reduction programs model and compensate for using a falloff factor similar to an overall $B$-factor $\left(\right.$ SCALEPACK $^{21}$ ) or by resolution-dependent scale factors [XDS/XSCALE, ${ }^{25}$ SCALA (CCP4)]. A similar approach may be expected for SFX data. A loss of electrons that is evenly distributed over the whole unit cell would result in an overall decrease of scattering power in the Bragg peaks. Analogously, an uncorrelated disorder in the crystalline lattice would result in an increase in the effective Wilson $B$-factor and diffuse scattering. ${ }^{26}$ Therefore, we scaled the intensities of the different data sets in the standard way, established by Blake and Phillips ${ }^{27}$ using a Wilson $B$-factor and an experimental linear scale factor $\mathrm{k}$ 


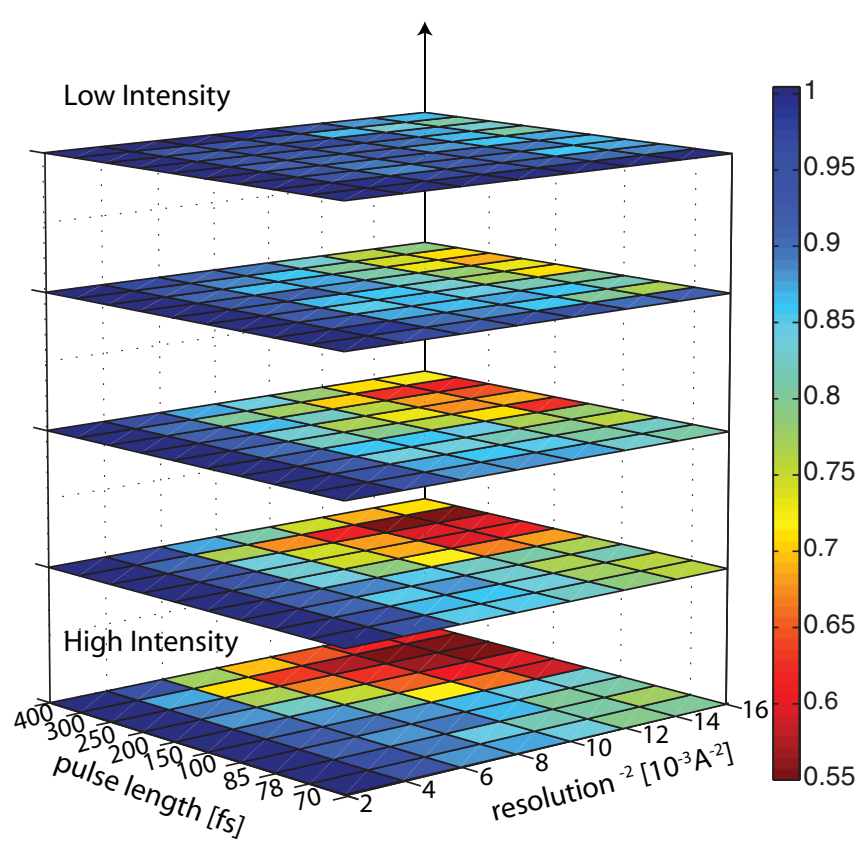

FIG. 3. (Color) Comparison of powder pattern intensities for different pulse durations and diffraction pattern intensities. Diffraction intensities of all pulse lengths and scattered intensity classes were treated as in Fig. 2(d). Intensities of all classes (different layers) and all pulse lengths are compared to the 70 fs data set of the lowest intensity class. The color encodes relative intensity.

(Table I). This assumed that the effect of the time dependence of the B factor could be neglected, which appears a reasonable first-order approximation. ${ }^{23}$ However, despite this correction, a clear trend remains apparent: the $R$-factor between data sets increase when the pulse length difference between data sets increases. Again, the $400 \mathrm{fs}$ data set remained an exception due to the low pulse energy [Ref. 23, Fig. S6(a)]. Along the same lines and within experimental error, the $R$-factors between FEL data and synchrotron powder data showed strong resolution dependence whereas a much smaller one was observed between the different FEL data sets [Ref. 23, Fig. S6(b)]. Changes due to radiation damage that can not be corrected by parametric scaling with a few variables would be those rooted in nonuniform modifications of the electron density of the unit cell.

Radiation damage under the experimental conditions of irradiance is a complex process. The primary effect is $K$-shell photoionization of the atoms in the molecules. Within 5$10 \mathrm{fs}$, an excited low- $Z$ atom relaxes through Auger decay. Auger- and photoelectrons generate electron cascades by electron-impact ionization. Collective dynamics have been predicted as ultrafast charge migration toward the center after "immediate" field ionization, ${ }^{28}$ then resulting in a quasineutral core and a positively charged outer layer that may peel off by Coulomb repulsion. It has thus been proposed to embed the sample of interest within a "sacrificial tamper," leading to slower expansion dynamics of the sample. ${ }^{29}$ In our experiment, the nanocrystals were surrounded by a water stream that served as a tamper. Water ionizes quickly due to the high cross sections for x-ray photoabsorption of oxygen and electron-impact ionization of hydrogen, efficiently providing electrons to neutralize the proposed positive center of the sample via charge migration. ${ }^{29}$ These predicted ionization and thermalization processes are difficult to quantify. They affect the x-ray-scattering properties of the sample on several partly interdependent levels.

First, core ionization and Auger relaxation change the orbital occupancy and the "effective" atomic number $Z(t)$ according to $f(q, t)=f(q, 0) Z(t) / Z,{ }^{29}$ and modify the $q$ dependence of the atomic form factors $f(q)$ in an exponential $q^{2}$-dependent manner. ${ }^{30}$ Second, ionization is a stochastic process leading to time-dependent changes in the electron density distribution $\rho(t)$ that are averaged over all molecules analyzed, making damage apparently homogeneous. However, the different cross sections of the elements, the nonhomogeneous distribution of elements in molecules and the discrete distribution of molecules in the unit cell may result in an inhomogeneous damage response. Third, the damage-induced (most likely inhomogeneous ${ }^{31}$ ) movements of atoms and ions in the molecule reduce the order of the crystalline lattice, resulting in a decrease in the intensity of Bragg peaks and an increase in diffuse scattering (Ref. 26, Fig. S8 in Ref. 23).

The high-resolution part of the diffraction pattern is the most sensitive to even small disorder and therefore is affected first and accumulates less intensity compared to the low-resolution region (Ref. 23, Fig. S5, Ref. 32). Indeed, data sets collected with varying pulse lengths (Fig. 2) or incident irradiance (Fig. 3) differed in the ratio of lowto-high-resolution Bragg intensities across the measured $q$ range, showing dose- and dose-rate-dependent effects. Unlike conventional crystallography in which damage usually accumulates during the collection of several-to-many diffraction images, allowing extrapolation of individual intensities to their zero-dose value, ${ }^{33}$ in SFX, the damage accumulates on the femtosecond time scale within the single exposure possible for a given crystal.

It has been suggested that consideration of the average statistical properties of the ionization process may allow recovery of the diffraction intensity of the undamaged molecule in case of identical atom types while a sample consisting of different elements is more difficult ${ }^{29}$ due to the uneven and structuredependent modification to the diffraction data, preventing simple rescaling ${ }^{34}$ (see also Ref. 23, Fig. S7). Computations have shown recently that the molecular electron density can be reconstructed when using an explicit damage model that predicts the electronic effects in the absence of nuclear motion and Auger effects by using 5 fs pulses. ${ }^{34}$ This, together with recent calculations on hollow atoms ${ }^{35}$ and our findings, suggests that very short pulses are required for high-resolution structure determination of nanocrystals at high doses. With the recent commissioning of further end stations at LCLS, providing $1.3 \AA$ wavelength, higher-resolution studies will be feasible in the near future, allowing to study the structural correspondent of the radiation-damage effects with atomic resolution. Nevertheless, since our detailed experimental analysis includes the intensity of each individual Bragg peak as a function of pulse duration and pulse energy (see Ref. 23), it allows for the comparison of predicted displacements and thus the magnitude of the change of intensity of the individual Bragg peaks based on calculations using an atomic model of lysozyme. This should allow for the testing of different, 
specific damage models and the evaluation of their accuracy by comparing experimental and calculated Bragg intensities one by one. This exceeds the possibilities of a global Wilson-type analysis based on general mathematical considerations using electron-density distributions based on the compositions of $\mathrm{C}$, $\mathrm{O}, \mathrm{N}, \mathrm{H}$, and $\mathrm{S}$, found generally in proteins. Our experiments were performed under conditions resulting in a very high dose ( 3 GGy), which might be difficult to achieve in the near future using hard x-rays. Eventually, however, such high doses are likely to be necessary for high-resolution studies of nanometersized crystals of bigger proteins consisting only of a small number of unit cells. Thus, despite the low resolution available, it is foreseeable that our data will be useful for quite some time to test specific models of damage occurring at very high dose.

Experiments were carried out at the Linac Coherent Light Source, a national user facility operated by Stanford University on behalf of the US Department of Energy, Office of Basic
Energy Sciences and at the Swiss Light Source, beamline X10SA, Paul Scherrer Institute, Villigen, Switzerland. We acknowledge support from the Max Planck Society for funding the development and operation of the CAMP instrument within the ASG at CFEL, the Helmholtz Association, CBST at UC Davis, DOE Grant No. DE-SC0002141, the National Science foundation NSF Grants No. 0417142 and No. MCB-1021557, the National Institute of Health (Grants No. 1R01GM09558301 and No. 1U54GM094625-01), the U.S. Department of Energy through the PULSE Institute at the SLAC National Accelerator Laboratory and Lawrence Livermore National Laboratory under Contract No. DE-AC52-07NA27344, and the Joachim Herz Stiftung. We thank Ulrike Mersdorf for technical support, Andreas Menzel for helpful discussions and his scripts, and Clemens Schulze-Briese and Fabia Gozzo for access to the powder-diffraction endstation at the SLS. We especially thank the staff of the LCLS for their outstanding facility and support in carrying out these experiments.
*Present address: European XFEL GmbH, Albert Einstein Ring 19, DE-22607 Hamburg, Germany.

†Ilme.Schlichting@mpimf-heidelberg.mpg.de

${ }^{1}$ J. Holton and K. Frankel, Acta Crystallogr., Sect. D: Biol. Crystallogr. 66, 393 (2010).

${ }^{2}$ M. Hoener, L. Fang, O. Kornilov, O. Gessner, S. Pratt, M. Guehr, E. Kanter, C. Blaga, C. Bostedt, J. Bozek, P. H. Bucksbaum, C. Buth, M. Chen, R. Coffee, J. Cryan, L. DiMauro, M. Glownia, E. Hosler, E. Kukk, S. R. Leone, B. McFarland, M. Messerschmidt, B. Murphy, V. Petrovic, D. Rolles, and N. Berrah, Phys. Rev. Lett. 104, 253002 (2010).

${ }^{3}$ L. Young, E. Kanter, B. Kraessig, Y. Li, A. March, S. Pratt, R. Santra, S. Southworth, N. Rohringer, L. DiMauro, G. Doumy, C. A. Roedig, N. Berrah, L. Fang, M. Hoener, P. H. Bucksbaum, J. P. Cryan, S. Ghimire, J. M. Glownia, D. A. Reis, J. D. Bozek, C. Bostedt, and M. Messerschmidt, Nature (London) 466, 56 (2010).

${ }^{4}$ B. Ziaja, A. de Castro, E. Weckert, and T. Moeller, Eur. Phys. J. D 40, 465 (2006).

${ }^{5}$ P. Persson, S. Lunell, A. Szoeke, B. Ziaja, and J. Hajdu, Protein Sci. 10, 2480 (2001).

${ }^{6}$ W. Burmeister, Acta Crystallogr., Sect. D: Biol. Crystallogr. 56, 328 (2000).

${ }^{7}$ R. Ravelli and S. McSweeney, Structure 8, 315 (2000).

${ }^{8}$ I. Schlichting, J. Berendzen, K. Chu, A. Stock, S. Maves, D. Benson, R. Sweet, D. Ringe, G. Petsko, and S. Sligar, Science 287, 1615 (2000).

${ }^{9}$ G. Berglund, G. Carlsson, A. Smith, H. Szoeke, A. Henriksen, and J. Hajdu, Nature (London) 417, 463 (2002).

${ }^{10}$ R. Owen, E. Rudino-Pinera, and E. Garman, Proc. Natl. Acad. Sci. USA 103, 4912 (2006).

${ }^{11}$ R. Henderson, Q. Rev. Biophys. 28, 171 (1995).

${ }^{12}$ R. Neutze, R. Wouts, D. van der Spoel, E. Weckert, and J. Hajdu, Nature (London) 406, 752 (2000).

${ }^{13}$ P. Emma, R. Akre, J. Arthur, R. Bionta, C. Bostedt, J. Bozek, A. Brachmann, P. Bucksbaum, R. Coffee, F. Decker, Y. Ding, D. Dowell, S. Edstrom, A. Fisher, J. Frisch, S. Gilevich, J. Hastings, G. Hays, Ph. Hering, Z. Huang, R. Iverson, H. Loos,
M. Messerschmidt, A. Miahnahri, S. Moeller, H.-D. Nuhn, G. Pile, D. Ratner, J. Rzepiela, D. Schultz, T. Smith, P. Stefan, H. Tompkins, J. Turner, J. Welch, W. White, J. Wu, G. Yocky, and J. Galayda, Nat. Photonics 4, 641 (2010).

${ }^{14}$ H. Chapman, P. Fromme, A. Barty, T. White, R. Kirian, A. Aquila, M. Hunter, J. Schulz, D. DePonte, U. Weierstall, R. B. Doak, F. R. N. C. Maia, A. V. Martin, I. Schlichting, L. Lomb, N. Coppola, R. L. Shoeman, S. W. Epp, R. Hartmann, D. Rolles, A. Rudenko, L. Foucar, N. Kimmel, G. Weidenspointner, P. Holl, M. Liang, M. Barthelmess, C. Caleman, S. Boutet, M. J. Bogan, J. Krzywinski, C. Bostedt, S. Bajt, L. Gumprecht, B. Rudek, B. Erk, C. Schmidt, A. Hömke, C. Reich, D. Pietschner, L. Strüder, G. Hauser, H. Gorke, J. Ullrich, S. Herrmann, G. Schaller, F. Schopper, H. Soltau, K.-U. Kühnel, M. Messerschmidt, J. D. Bozek, S. P. Hau-Riege, M. Frank, C. Y. Hampton, R. G. Sierra, D. Starodub, G. J. Williams, J. Hajdu, N. Timneanu, M. M. Seibert, J. Andreasson, A. Rocker, O. Jönsson, M. Svenda, S. Stern, K. Nass, R. Andritschke, C.-D. Schröter, F. Krasniqi, M. Bott, K. E. Schmidt, X. Wang, I. Grotjohann, J. M. Holton, T. R. M. Barends, R. Neutze, S. Marchesini, R. Fromme, S. Schorb, D. Rupp, M. Adolph, T. Gorkhover, I. Andersson, H. Hirsemann, G. Potdevin, H. Graafsma, B. Nilsson, and J. C. H. Spence, Nature (London) 470, 73 (2011).

${ }^{15}$ The protein lysozyme $\left(\mathrm{C}_{613} \mathrm{H}_{959} \mathrm{~N}_{193} \mathrm{O}_{185} \mathrm{~S}_{10}, 3.2-\mathrm{nm}\right.$ diameter forms loosely packed crystals containing large solvent channels $(38 \% \mathrm{~V} / \mathrm{V})$.

${ }^{16}$ J. Bozek, Eur. Phys. J.-Spec. Top. 169, 129 (2009).

${ }^{17}$ L. Strueder, S. Epp, D. Rolles, R. Hartmann, P. Holl, G. Lutz, H. Soltau, R. Eckart, C. Reich, K. Heinzinger, C. Thamm, A. Rudenko, F. Krasniqi, K.-U. Kühnel, C. Bauer, C.-D. Schröter, R. Moshammer, S. Techert, D. Miessner, M. Porro, O. Hälker, N. Meidinger, N. Kimmel, R. Andritschke, F. Schopper, G. Weidenspointner, A. Ziegler, D. Pietschner, S. Herrmann, U. Pietsch, A. Walenta, W. Leitenberger, C. Bostedt, T. Möller, D. Rupp, M. Adolph, H. Graafsma, H. Hirsemann, K. Gärtner, R. Richter, L. Foucar, R. L. Shoeman, I. Schlichting, and J. Ullrich, Nucl. Instrum. Meth. A 614, 483 (2010).

${ }^{18}$ D. DePonte, U. Weierstall, K. Schmidt, J. Warner, D. Starodub, J. Spence, and R. Doak, J. Phys. D: Appl. Phys. 41, 195505, (2008). 
${ }^{19}$ J. Falkner, M. Ali, J. Jamison, J. Zhang, S. Adrianse, R. Simpson, M. Calabretta, W. Radding, G. Phillips Jr., and V. Colvin, Chem. Mater. 17, 2679 (2005)

${ }^{20}$ K. Paithankar, R. Owen, and E. Garman, J. Synchrotron Radiat. 16, 152 (2009).

${ }^{21}$ Z. Otwinowski and W. Minor, Methods Enzymol. 276, 307 (1997).

${ }^{22}$ Q. Liu, A. Weaver, T. Xiang, D. Thiel, and Q. Hao, Acta Crystallogr., Sect. D: Biol. Crystallogr. 59, 1016 (2003).

${ }^{23}$ See Supplemental Material at http://link.aps.org/supplemental/ 10.1103/PhysRevB.84.214111 for further figures and supplemental material concerning the analysis. The powder pattern intensities are also deposited.

${ }^{24}$ C. Caleman, G. Huldt, F. Maia, C. Ortiz, F. Parak, J. Hajdu, D. van der Spoel, H. Chapman, and N. Timneanu, ACS Nano 5, 139 (2011).

${ }^{25}$ W. Kabsch, J. Appl. Crystallogr. 21, 916 (1988).
${ }^{26}$ B. Warren, X-Ray Diffraction (Dover Publications, New York, 1990).

${ }^{27}$ C. Blake and D. Phillips, Vienna: International Atomic Energy Agency, 183 (1962).

${ }^{28}$ C. Gnodtke, U. Saalmann, and J. M. Rost, Phys. Rev. A 79, 041201 (2009).

${ }^{29}$ S. P. Hau-Riege, R. A. London, H. N. Chapman, A. Szoke, and N. Timneanu, Phys. Rev. Lett. 98, 198302 (2007).

${ }^{30}$ S. P. Hau-Riege, Phys. Rev. A 76, 042511 (2007).

${ }^{31}$ Z. Jurek and G. Faigel, Europhys. Lett. 86, 68003 (2009).

${ }^{32}$ A. Barty, Nature Photonics (accepted, 2011).

${ }^{33}$ K. Diederichs, S. McSweeney, and R. Ravelli, Acta Crystallogr., Sect. D: Biol. Crystallogr. 59, 903 (2003).

${ }^{34}$ H. Quiney and K. Nugent, Nat. Phys. 7, 142 (2010).

${ }^{35}$ S. K. Son, L. Young, and R. Santra, Phys. Rev. A 83, 033402 (2011). 